\title{
Giant vesicles: a powerful tool to reconstruct bacterial division assemblies in cell-like compartments
}

\author{
Mercedes Jiménez, ${ }^{1 *}$ Ariadna Martos, ${ }^{2}$ \\ Elisa J. Cabré, ${ }^{1}$ Ana Raso ${ }^{1,2}$ and Germán Rivas ${ }^{1 *}$ \\ ${ }^{1}$ Centro de Investigaciones Biológicas, CSIC, c/Ramiro \\ de Maeztu 9, 28040 Madrid, Spain. \\ ${ }^{2}$ Max Planck Institute of Biochemistry, Am Klopferspitz \\ 18, D-82152, Martinsried, Germany.
}

\section{Summary}

The use of artificial lipid membranes, structured as giant unilamellar vesicles (GUVs), provides the opportunity to investigate membrane-associated biological processes under defined experimental conditions. Due to their large size, they are uniquely adapted to investigate the properties and organization (in time and space) of macromolecular complexes incorporated in the vesicle interior by imaging and micro-spectroscopic techniques. Experimental methods to produce giant vesicles and to encapsulate proteins inside them are here reviewed. Previous experimental work to reconstitute elements of the bacterial division machinery in these membrane-like systems is summarized. Future challenges towards reconstructing minimal divisome assemblies in giant vesicles as cytomimetic containers are discussed.

\section{Introduction}

One of the current goals of research in biosciences is to reconstruct in the test tube, with the minimal set of elements, macromolecular assemblies forming part of functional cellular subsystems. This experimental challenge, which will help to reduce the gap between our current understanding of the relationship between molecular and cellular studies, requires integration of biochemical, biophysical, genetic, imaging and technological expertise (Liu and Fletcher, 2009; Martos et al., 2012a; Monterroso et al., 2013; Schwille, 2013). This strategy complements

Received 22 March, 2013; revised 10 July, 2013; accepted 11 July, 2013. *For correspondence. E-mail enoe@cib.csic.es; grivas @ cib.csic.es; Tel. (+34) 918373112; Fax (+34) 915360432. Special Issue - Reconstructing the essential bacterial cell cycle machinery. other synthetic efforts aiming at (i) the achievement of minimal cells, by eliminating certain genes and metabolic pathways (Nandagopal and Elowitz, 2011) and (ii) the engineering of new systems, or of biological functions, that do not exist in nature (Endy, 2005). In this paper, we will focus on the first of these synthetic approaches emphasizing how it can be used to understand fundamental problems of biological assembly and self-organization, namely how elements interact in a dynamic manner to yield functional high-order entities forming part of the bacterial division machinery.

Cytokinesis is an essential process that in bacteria is understood in sufficient detail as most of the division elements, their functions, and a large part of their biochemical activities are relatively well known (Adams and Errington, 2009; Lutkenhaus et al., 2012; Egan and Vollmer, 2013). In Escherichia coli, the cell division protein FtsZ is anchored to the cytoplasmic membrane by the action of the membrane protein ZipA and the amphitropic protein FtsA, forming the initial molecular assembly of the division machinery, the proto-ring. The proto-ring drives cytokinesis at midcell, where FtsZ polymers formed upon GTP binding arrange in a dynamic Z-ring active in division (Vicente and Rico, 2006; Vicente et al., 2006). Positioning of FtsZ at midcell is directed by the Min proteins that oscillate from pole to pole, blocking the polymerization of FtsZ at both cell poles, and by the nucleoid occlusion that prevents FtsZ polymer formation as long as the nucleoid is not segregated. The reader is referred to recent comprehensive reviews on this topic for further information (Erickson et al., 2010; Mingorance et al., 2010; Lutkenhaus et al., 2012; Egan and Vollmer, 2013).

Most of the key macromolecular reactions in bacterial division take place at membrane surfaces. For this reason, a considerable effort is being done to reconstitute and study the activity, assembly and interactions of essential division proteins (e.g. FtsZ) in model membranes (reviewed in Martos et al., 2012a and Rivas et al., 2013). Among them, giant unilamellar vesicles, because of their large size well above the optical resolution limit (from 10 to $100 \mu \mathrm{m}$ ), are particularly well adapted to 
reconstruct minimal division assemblies as, in principle, the organization of these dynamic assemblies, and their modulation in time and space, can be monitored inside them using high-resolution fluorescence microscopy and single-molecule imaging techniques (Schwille and Diez, 2009; Martos et al., 2012a). Since the development of the first reproducible procedure for giant vesicles production (Reeves and Dowben, 1969), these methods have been extensively used to study the biochemical and biophysical mechanisms linked to the formation of lipid membrane domains - including lipid rafts (reviewed in Schwille and Diez, 2009), and of vesicle fusion and scission - such as membrane tubulation and budding (Baumbart et al., 2011; Terasawa et al., 2013). Moreover, GUVs have been instrumental to study the influence of protein motors and fibres on the mechanical properties of lipid vesicles (Kale et al., 2010; Stachowiak et al., 2010; 2012) and have also been used as a cell-like container for cell-free gene expression inside vesicles (Noireaux et al., 2011; Stano and Luisi, 2013). For a more comprehensive review on giant vesicles, the reader is referred to Walde et al., 2010.

The purpose of the present paper is to provide a summary of the experimental implementation in the production of giant vesicles (with emphasis on methods well adapted for the encapsulation of biological macromol- ecules inside the vesicles), to summarize results in previously published experimental studies of reconstitution of bacterial division elements in giant vesicles and to present future developments and challenges towards reconstructing minimal division assemblies in these cell-like containers. Below, we revised the main methods and outstanding results in the field.

\section{Methods based in lipid hydration}

The first technique described to obtain micrometre-size lipid unilamellar vesicles involves the swelling or hydration of a dry (or semi dry) film of lipid (amphiphilic) molecules that, upon hydration, spontaneously selforganizes to form a closed lipid compartment (Fig. 1A) (Reeves and Dowben, 1969). The procedure is simple and requires minimal sample manipulation, allowing the use of a broad variety of buffer compositions, and practically all kinds of lipids, including native membranes. The formation of multilamellar vesicles can be minimized by using the appropriate amount of lipids and by controlling both the uniformity of the deposited lipid layers and the hydration conditions. However, the efficiency of macromolecular entrapment is low, and the vesicles produced by this technique are very polydispersed in size (Table 1).

Table 1. Summary of the selected methods for giant vesicles production. ${ }^{a}$

\begin{tabular}{|c|c|c|c|c|c|}
\hline Method & Yield $^{\mathrm{b}}$ & Size distribution & $\begin{array}{l}\text { Expected } \\
\text { encapsulation } \\
\text { level }\end{array}$ & Comments & Ref. \\
\hline Natural swelling & High & Polydisperse & Low & $\begin{array}{l}\text { - Simple } \\
\text { - Suitable for lipid and native } \\
\text { membranes }\end{array}$ & $\begin{array}{l}\text { (Reeves and Dowben, } \\
\text { 1969; Dominak and } \\
\text { Keating, 2008; Horger } \\
\text { et al., 2009; Tsai et al., } \\
\text { 2011) }\end{array}$ \\
\hline Electroformation & $\begin{array}{l}\text { Moderate/ } \\
\text { High }\end{array}$ & $\begin{array}{l}\text { Polydisperse, more } \\
\text { controlled }\end{array}$ & Low & $\begin{array}{l}\text { - Simple equipment } \\
\text { - Suitable for lipid and native } \\
\text { membranes }\end{array}$ & $\begin{array}{l}\text { (Angelova and Dimitrov, } \\
\text { 1986, 1992; Merkle et al., } \\
\text { 2008; Pott et al., 2008; } \\
\text { Montes et al., 2010; } \\
\text { Stachowiak et al., 2012) }\end{array}$ \\
\hline Droplet transfer & High & $\begin{array}{l}\text { Polydisperse, more } \\
\text { controlled }\end{array}$ & High & $\begin{array}{l}\text { - Simple } \\
\text { - Possible presence of oil inside } \\
\text { vesicles }\end{array}$ & $\begin{array}{l}\text { (Pautot et al., 2003; Carrara } \\
\quad \text { et al., 2012) }\end{array}$ \\
\hline cDICE & High & $\begin{array}{l}\text { Monodispersity } \\
\text { Controllable size }\end{array}$ & High & $\begin{array}{l}\text { - Simple equipment } \\
\text { - Possible presence of oil inside } \\
\text { vesicles }\end{array}$ & (Abkarian et al., 2011) \\
\hline Microfluidic & High & $\begin{array}{l}\text { Monodispersity } \\
\text { Controllable size }\end{array}$ & High & $\begin{array}{l}\text { - Simple equipment } \\
\text { - High-throughput, highly } \\
\text { reproducible } \\
\text { - Very fast }\end{array}$ & (Ota et al., 2009) \\
\hline Jetting & High & $\begin{array}{l}\text { Large monodisperse } \\
\text { vesicles }\end{array}$ & High & $\begin{array}{l}\text { - Sophisticated equipment } \\
\text { - High-throughput } \\
\text { - Instable vesicles } \\
\text { - Very fast }\end{array}$ & $\begin{array}{l}\text { (Stachowiak et al., 2008; Li } \\
\text { et al., 2009) }\end{array}$ \\
\hline $\begin{array}{l}\text { Lipid-coated ice } \\
\text { droplets hydration }\end{array}$ & High & $\begin{array}{l}\text { Controlled } \\
\text { monodispersity }\end{array}$ & High & Simple equipment & $\begin{array}{l}\text { (Sugiura et al.,2008; } \\
\quad \text { Kuroiwa et al., 2012) }\end{array}$ \\
\hline
\end{tabular}

a. For a more comprehensive view on advantages and disadvantages of these methods the reader is referred to table 3 in Walde et al., 2010. b. Yield referred as number of vesicles. 
A

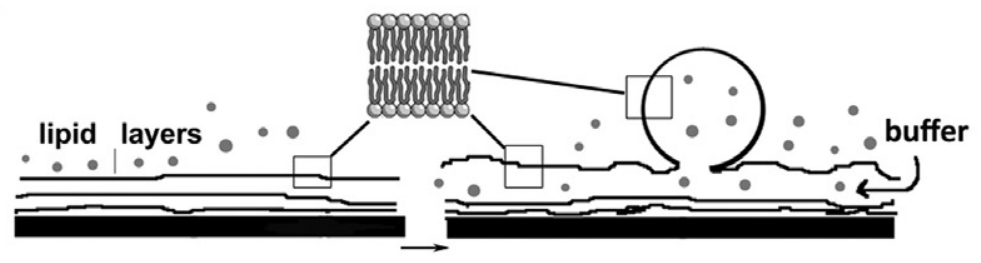

B

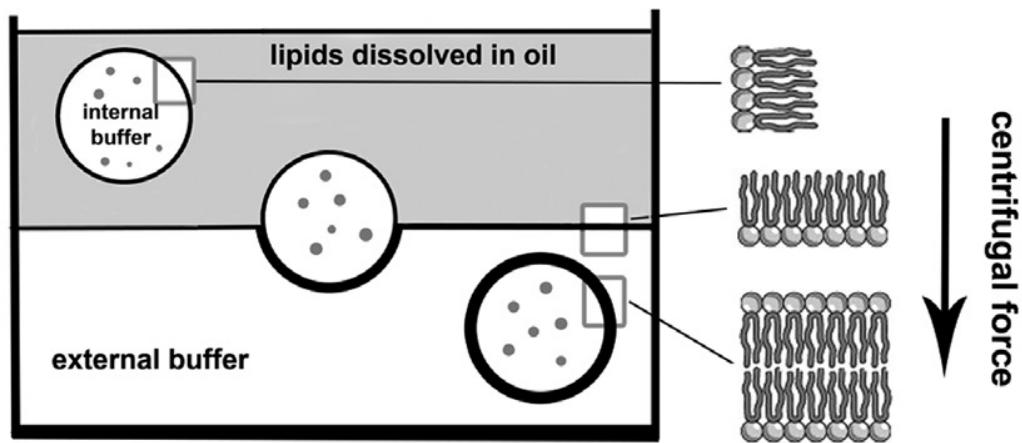

C

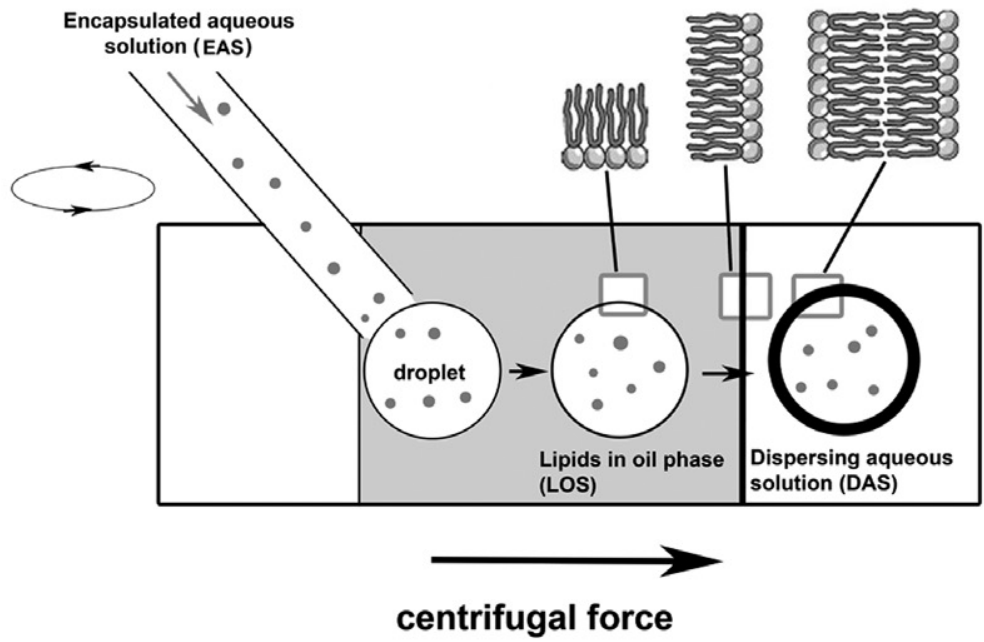

Fig. 1. Schematic representations of the main methods for the formation of giant vesicles.

A. Lipid hydration. Natural swelling and electroformation methods are based in the same principle: lipid layers deposited onto a solid surface render spherical structures upon hydration with the buffer solution. B. Droplet transfer. Aqueous droplets containing the internal buffer are coated by lipids in the lipid-stabilized oil phase. The migration through the lipid-saturated phase leads to the stabilization of vesicles that will be recovered from the external buffer phase. C. Continuous Droplet Interface Crossing Encapsulation method (cDICE). Droplets are produced when the encapsulated aqueous solutions (EAS) are detached from the capillary while both lipid-in-oil solution (LOS) and EAS are subjected to a centrifugal force in a Petri dish. Droplets enter the LOS phase and lipids coat them. Driven by the centrifugal force, lipid-coated droplets cross the LOS-DAS interface giving bilayer-coated vesicles.
The size homogeneity of the giant vesicles produced by swelling can be improved if the hydration of the lipids is done in the presence of a controlled electrical field, either between two platinum wires or two metal-coated surface glasses (usually using indium tin oxide, as the metal component) (Angelova and Dimitrov, 1986; Angelova et al., 1992). Although the vesicle yield is moderate (Table 1), this is one of the most reliable protocols to produce giant vesicles made from lipid mixtures, which usually remain attached to the surface of support allowing their observation during extended periods of time and the manipulation of individual vesicles as well. The formation of giant vesicles in slowly alternating fields is usually more efficient when it is done in low-salt buffers, although recent developments have described vesicle production under physiological ionic conditions (Pott et al., 2008; Montes et al., 2010). Electroformed vesicles have therefore been widely used to investigate physicochemical properties of lipid mixtures, including the formation of lipid domains linked to phase separation processes (Schwille, 2011). In addition, the analysis of lipid organization in vesicles made from native membranes has been described (Montes et al., 2010). Proteins incorporated in the outer face of electroformed vesicles have been found to influence 
membrane properties including vesicle deformation by the action of motor proteins (Liu et al., 2008) and membrane curvature changes coupled to high-protein surface densities (Stachowiak et al., 2012). Interestingly, the properties of encapsulated actin assemblies on electroformed giant vesicles, made in part from native membranes, have been described (Merkle et al., 2008). Recent technical and methodological improvements have partially overcome one of the main disadvantages of hydration-based methods, namely the low yield of encapsulation of biological macromolecules. Gentle hydration of an agarose hydrogel film infiltrated with lipids (Horger et al., 2009; Tsai et al., 2011) or co-encapsulation of solutions containing high concentrations of unrelated macromolecules (acting as crowders Zhou et al., 2008) (Dominak and Keating, 2008) have resulted in higher amounts of entrapped macromolecules at the vesicle lumen.

\section{Methods based on water/oil emulsions}

To enhance the efficiency of encapsulation of macromolecules, several protocols based in water-oil emulsion have been developed. One of them (droplet transfer or double emulsion method) involves the assembly of two phospholipid monolayers (independently produced) to form unilamellar vesicles (Pautot et al., 2003) (Fig. 1B). When mixing the aqueous solutes and an oil phase containing dispersed lipids, the later spontaneously reorganize to assemble the inner monolayer around the formed aqueous microdroplets. For the formation of the outer lipid monolayer, this emulsion is placed on top of a two-phase system, consisting of a lipid-containing oil (upper phase) and an aqueous solution (lower phase) where the interfacial region is saturated by lipids. As the emulsion droplets are heavier than the upper phase oil, they spontaneously move through the interface (where a bilayer is formed) towards the lower (aqueous) phase from where the vesicles - with the trapped solutes inside - are recovered, whereas the non-trapped solutes are absent in this final solution (Fig. 1B). Droplet migration from the oil-lipid phase can be accelerated by low centrifugal forces and/or by using droplets containing sucrose in an equi-osmotic glucose-containing lower phase (Carrara et al., 2012). The size of the vesicles formed is heterogeneous as it depends on the size of the droplets formed during the emulsion step. These vesicles are well suited for biochemically controlled reconstitution studies and for bioreactor applications as proteins (and other macromolecular assemblies) can be entrapped with high efficiency, and the vesicles are free of non-trapped macromolecules around them. A potential drawback of this technique is that oil traces cannot be ruled out, requiring specific control experiments to check that the properties of entrapped macromolecules are not affected.
A variant of the double emulsion technique, based on a continuous dripping of droplets off a capillary forcing their passage through a lipid-oil/aqueous interface by a centrifugal force, has been recently developed (Abkarian et al., 2011). One of the main advantages of the continuous droplet interface crossing encapsulation (CDICE) method is that the produced vesicles are essentially monodisperse as their size is controlled by the diameter of the capillary tip (Fig. 1C). In this protocol, a dispersing aqueous solution (DAS, the final outer buffer), and a lipidin-oil solution (LOS) are centrifuged on a Petri dish. The aqueous solution (EAS), containing the macromolecules to be incorporated, is injected into the LOS solution through a capillary while the dish is rotating. The DAS and LOS solutions are not miscible, leading to an interface perpendicular to the centrifugal force, which favours the formation of unilamellar vesicles when the droplets of EAS are forced through the lipid interface. Since centrifugal is the main driving force, the droplets with the encapsulated solution must have a higher density than the dispersing solution, which in turn needs to have a higher density than the lipid-in-oil solution. This protocol produces, in the order of minutes and in a single step, a suspension of monodisperse vesicles whose size can be controlled, with high-encapsulation efficiency.

\section{Microfluidic approaches}

Microfluidic-based methods are, in principle, uniquely adapted to produce, in large scale, highly monodisperse giant vesicles with a good yield of macromolecular incorporation in their interior (Stachowiak et al., 2008; Ota et al., 2009; Matosevic, 2012). Microfluidic platforms allow handling and controlling fluids in a micrometric scale, which are characterized by a laminar flow that is ultimately determined by microscopic physical properties (such as speed, viscosity and capillary diameter) whose values do not correspond to those measured at the macroscopic scale. The flow can be either continuous, from microfluidics channels (continuous-flow microfluidics), or discontinuous, using digitally controlled picoliter droplet compartments (droplets in microfluidics). Both flow systems have been already applied to produce giant vesicles in devices fabricated by standard soft-lithographic techniques, using mainly polydimethylsiloxane, polymers, silicon or glass. For a more comprehensive review on the application of microfluidics technologies to the production of giant vesicles, the reader is referred to Matosevic (2012). Here, the variants of this technology potentially more adapted for biological reconstitution studies will be briefly described.

The production of giant vesicles by flowing the solution containing the macromolecules against a lipid bilayer previously formed at a T-junction device perpendicular to the 
A
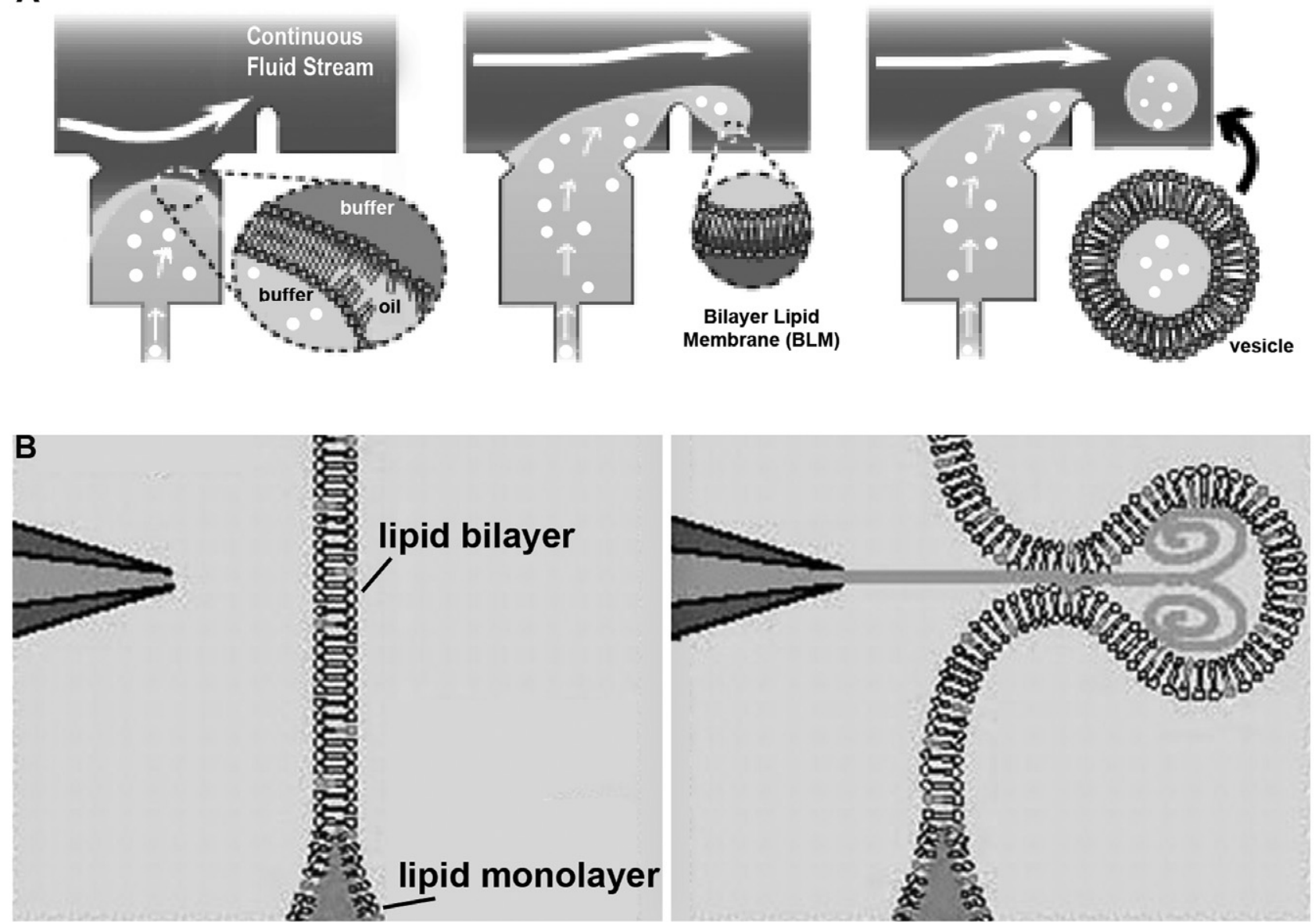

Fig. 2. Microfluidic approaches for the generation of unilamellar vesicles.

A. Main steps of the microfluidic formation of vesicles in a T-junction device (adapted from Ota et al., 2009). A cross flow at the microfluidic T-junction drives the contact of monolayers to form a bilayer. The outward flow bends out the bilayer and the fission of the leading edge generates vesicles.

B. Microfluidic jetting principle to obtain filled vesicles blowing the aqueous buffer and deforming a lipid bilayer. Reprinted with permission from Richmond, et al., 2011.

continuous flow has been recently described (Fig. 2A) (Ota et al., 2009). This method allows using sample volumes lower than $5 \mu \mathrm{l}$, a volume significantly reduced regarding previous microfluidics protocols, and a more precise control of the droplet size by modulating the flow. This method has been used to encapsulate a functional cell-free gene expression system inside vesicles and to investigate the membrane properties of the vesicles using the pore-forming protein haemolysin.

The formation of giant vesicles by a jet-blowing process, based on the operation of an inkjet printer, has been described (Stachowiak et al., 2008; Li et al., 2009). The jetting system is controlled by a piezoelectric device that injects the solution containing the macromolecules and deforms a lipid bilayer previously formed, producing hundreds of monodisperse vesicles per minute with a very high efficiency of macromolecule encapsulation (Fig. 2B).
The main drawbacks of this unique system relate with the need of sophisticated instrumentation, the relatively high lower limit of vesicle sizes formed (in the order of $50 \mu \mathrm{m}$ of diameter Li et al., 2009) and the possible presence of oil traces inside and at the membrane surface (Table 1).

The production of giant vesicles based on a water-inhexane emulsion formed in a microfluidic device, and stabilized by surfactants, has been developed (Sugiura et al., 2008; Matosevic, 2012). This technique (lipidcoated ice droplet hydration) which produces vesicles of controlled sizes (from $4 \mu \mathrm{m}$ and $20 \mu \mathrm{m}$ ) consists in four steps: emulsification, surfactant replacement, solvent evaporation, and, finally, hydration. Droplets are frozen and separated from the hexane solution by precipitation. Surfactants are replaced with phospholipids, and the droplets are hydrated to generate giant vesicles. Special requirements should be taken into account to avoid 

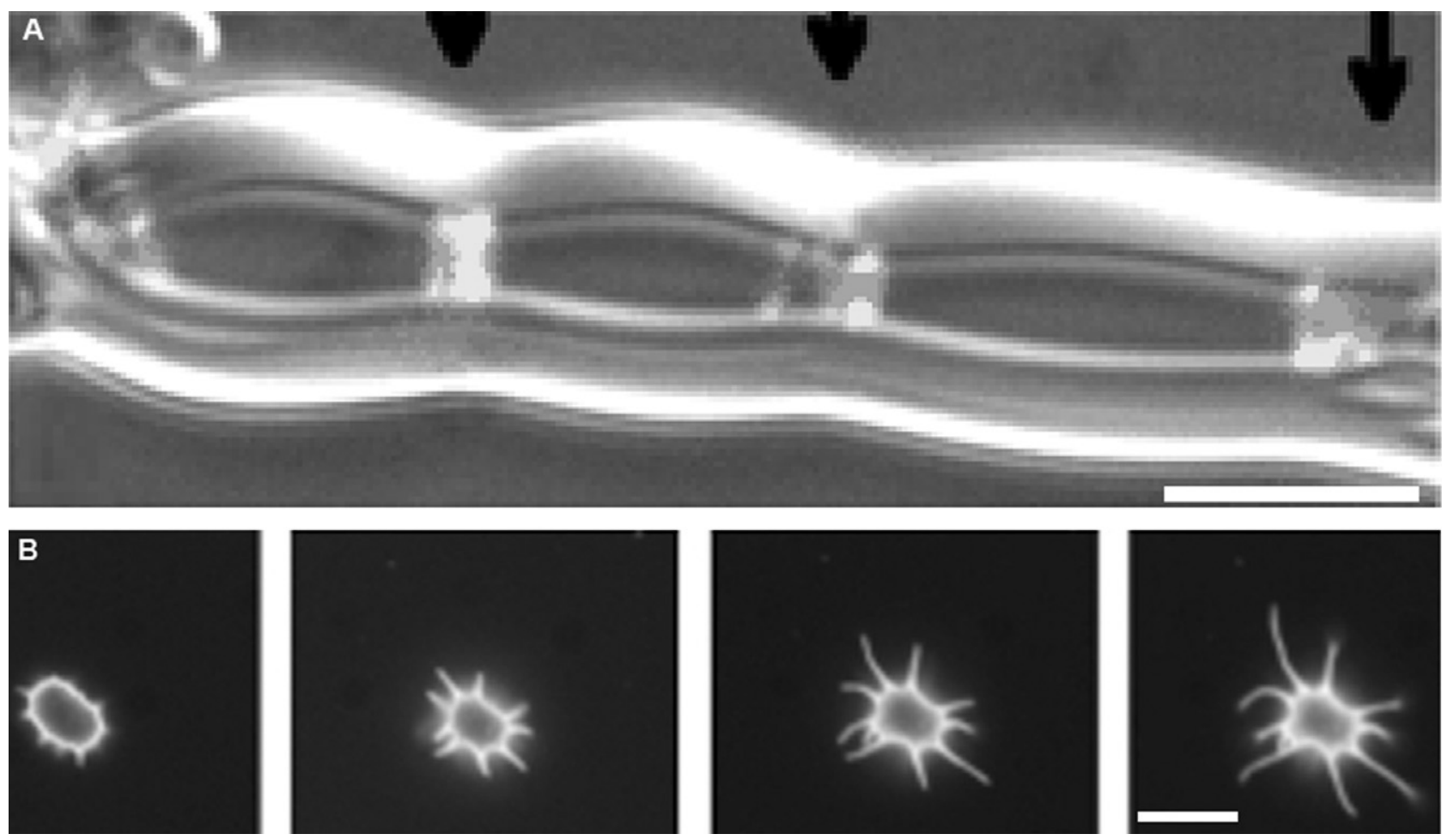

Fig. 3. FtsZ-mts polymerization in liposomes.

A. Liposomes were prepared from a mix (from $2 \%$ to $40 \%$ ) of DOPG (1,2-Dioleoyl- sn -Glycero-3- [Phospho-rac-(1-glycerol)]) and egg PC (phosphatidylcholine). Lipids were dissolved in methanol, mixed, dried and re-suspended at $10 \mathrm{mg} / \mathrm{ml}$ in water by vigorous vortexing. The suspension was placed on a $4 \mathrm{~cm}$ diameter Teflon disc and dried under an air current. The films were hydrated with five volumes of buffer and incubate for $2-3 \mathrm{~h}$ at $37^{\circ} \mathrm{C}$. Liposomes were then further suspended by gentle agitation, forming a turbid layer above the Teflon. After FtsZ-mts and GTP addition, FtsZ-mts protein, initially outside the liposomes, was spontaneously internalized into the liposomes and found assembling into rings and helical structures inside them. The fluorescent FtsZ is shown in yellow, superimposed on the differential interference contrast image of the liposome (Osawa et al., 2008).

B. The polymerization of this variant protein outside giant liposomes obtained with the method referred to above, originated membrane deformations visualized as tubules recorded in time-lapse images. (Osawa et al., 2009). Bars represent $5 \mu$ m. Reprinted with permission from Osawa et al. 2008 and Osawa et al. 2009.

sample damage upon freezing. The method has recently been used to measure the efficiency of solute entrapment and the activity of certain enzymes inside the vesicles (Kuroiwa et al., 2012).

\section{Insights on reconstructed membranes}

One major advantage of the reconstitution in these celllike membrane compartments is that both biochemical and biophysical parameters can, in principle, be controlled, allowing to study reactions between soluble and membrane-bound elements under relatively well-defined experimental conditions. These experimental approaches are relevant to investigate the activities, association and assembly properties of bacterial division elements, including their modulation in time and space, as many of the events involved in this essential process are membranelinked reactions.

Along these lines, an artificially membrane-attached FtsZ variant, which can bind to the membrane in the absence of the natural membrane anchors, the proteins FtsA and ZipA, was externally added to multilamellar liposomes formed using a modification of the hydration/ swelling protocol described above. These liposomes, after squeezing, may convert into tubular liposomes. Under these conditions, the membrane-bound Fts Z variant occasionally internalized into the liposome, and it was found at discrete regions producing a limited increase in the membrane curvature, which the authors have correlated with the formation of structures that mimic rings assimilated to Z-rings (Osawa et al., 2008) (Fig. 3A). The polymerization of FtsZ variant located at the outer face of liposomes produced lipid tubules and membrane deformations (Osawa et al., 2009; Osawa and Erickson, 2011) (Fig. 3B). Electron microscopy has revealed that polymers of the membrane-bound FtsZ variant are organized inside lipid tubes as ribbon-like structures (Milam et al., 2012) resembling the polymers formed by wild type FtsZ in the presence of high concentrations of crowders (González et al., 2003). 

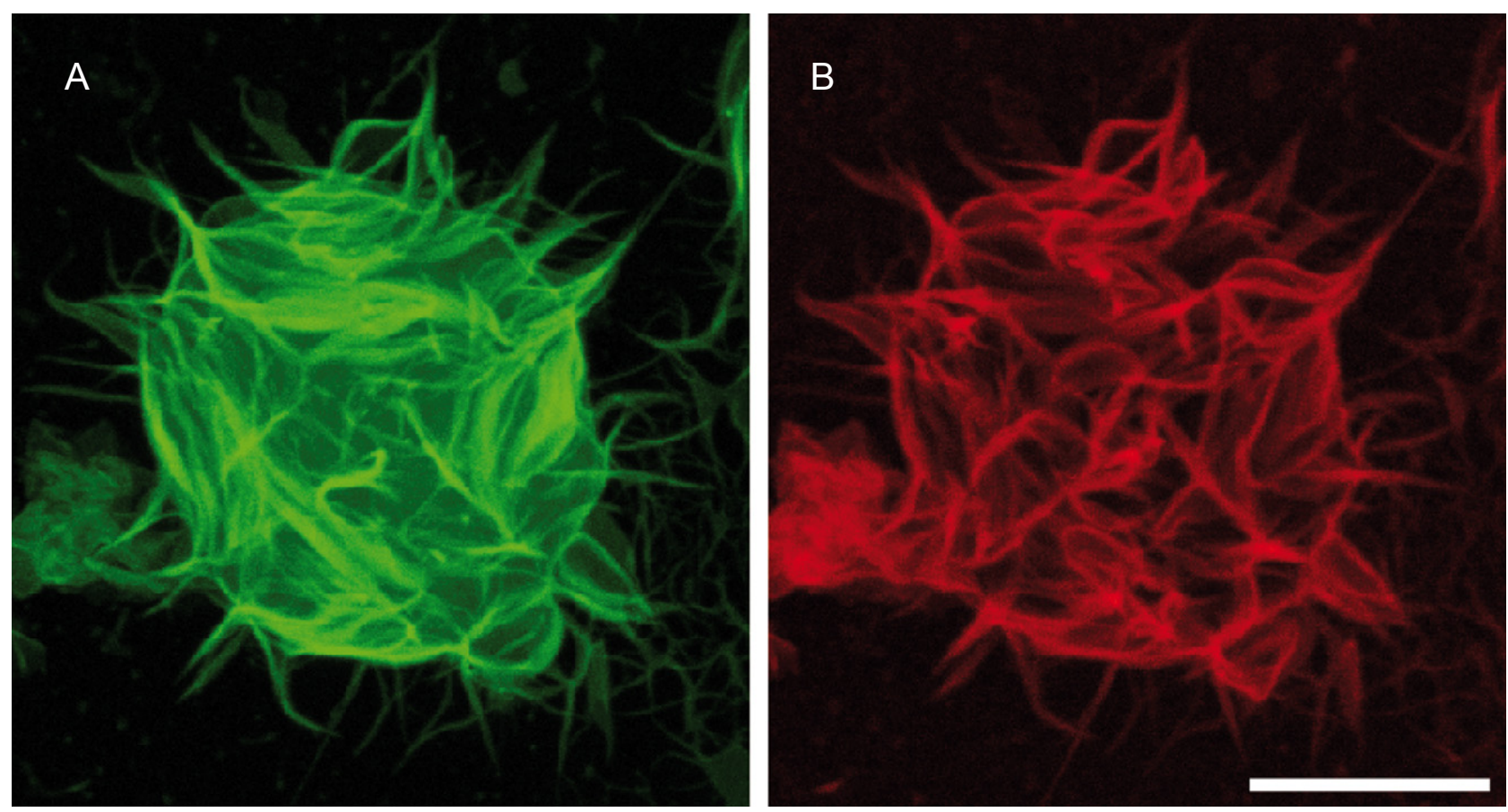

Fig. 4. Image analysis of sZipA-containing giant vesicle in the presence of GTP-FtsZ polymers.

A. Stack projection of cross-sectional confocal images of a sZipA-containing GUV in the presence of Alexa 488 labelled FtsZ and GTP.

B. Stack projection of cross-sectional confocal images of the same vesicle stained with the lipid dye DilC18. Giant vesicles were obtained by electroformation from the polar extract of $E$. coli lipids in the presence of high salt concentration following the method described in Pott et al. 2008. Original data (Martos, A., unpublished results). Bar represents $10 \mu \mathrm{m}$.

The polymerization of the natural FtsZ protein in the external face of ZipA-containing giant vesicles resulted in membrane deformation (Fig. 4) (Martos et al., 2012a), similar to the one observed with the membrane-bound FtsZ variants described above (Osawa et al., 2009). Wild type FtsZ and soluble ZipA, lacking the transmembrane region (sZipA), were fluorescently labelled after isolation maintaining the interaction domains intact. Electroformed lipid vesicles where sZipA was anchored in the outer face via a functionalized lipid (DOGS-NTA-Ni, 1,2-dioleoyl-snglycero-3-\{[N-(5-amino-1-carboxypentyl) iminodiacetic acid]succinyl\} nickel salt) were morphologically deformed in the presence of FtsZ polymers. This strategy may allow studying the effect of companion proteins (such as FtsA), inhibitors and physiological ligands on the activities and interactions of FtsZ.

The native form of FtsZ has been encapsulated together with FtsA (Martos et al., 2012b) onto giant vesicles made from whole inner membrane isolated from bacteria, which contains ZipA naturally incorporated, in the presence of high concentrations of unrelated macromolecules to reproduce natural crowding (Jiménez et al., 2011). While FtsA is found adsorbed to the inner face of the giant vesicles, either alone or in the presence of non-polymerized FtsZ, the polymerization resulting from co-encapsulation of FtsZ with GTP analogues results in
FtsA dislodgement and its association to the FtsZ polymers in the lumen, indicating that the FtsA-membrane interaction can be counteracted by the association of FtsA to assembled FtsZ (Jiménez et al., 2011) (Fig. 5). This study showed that FtsA alone is not enough to anchor FtsZ to the membrane indicating that a local increase of ZipA concentration is necessary, in accordance with previous in vivo studies showing $E$. coli needs both proteins to complete division [revised in (Vicente and Rico, 2006)]. This finding also suggested additional roles for FtsA, related to its amphitropic character, such as participation in signalling events during proto-ring assembly or the activation of late division events. Related to this, the interaction between FtsA and the late-assembling FtsN protein has been described (Vicente et al., 2006; Rico et al., 2010). Recently, it has been described a membrane dilation in lipid vesicles containing SZipA at both sides of the membrane when FtsZ polymerization was triggered by the release of caged GTP (Lopez-Montero et al., 2013).

\section{What next?}

In this review, we have described recent technologies to produce giant vesicles that are well adapted for the encapsulation of proteins and molecular assemblies 


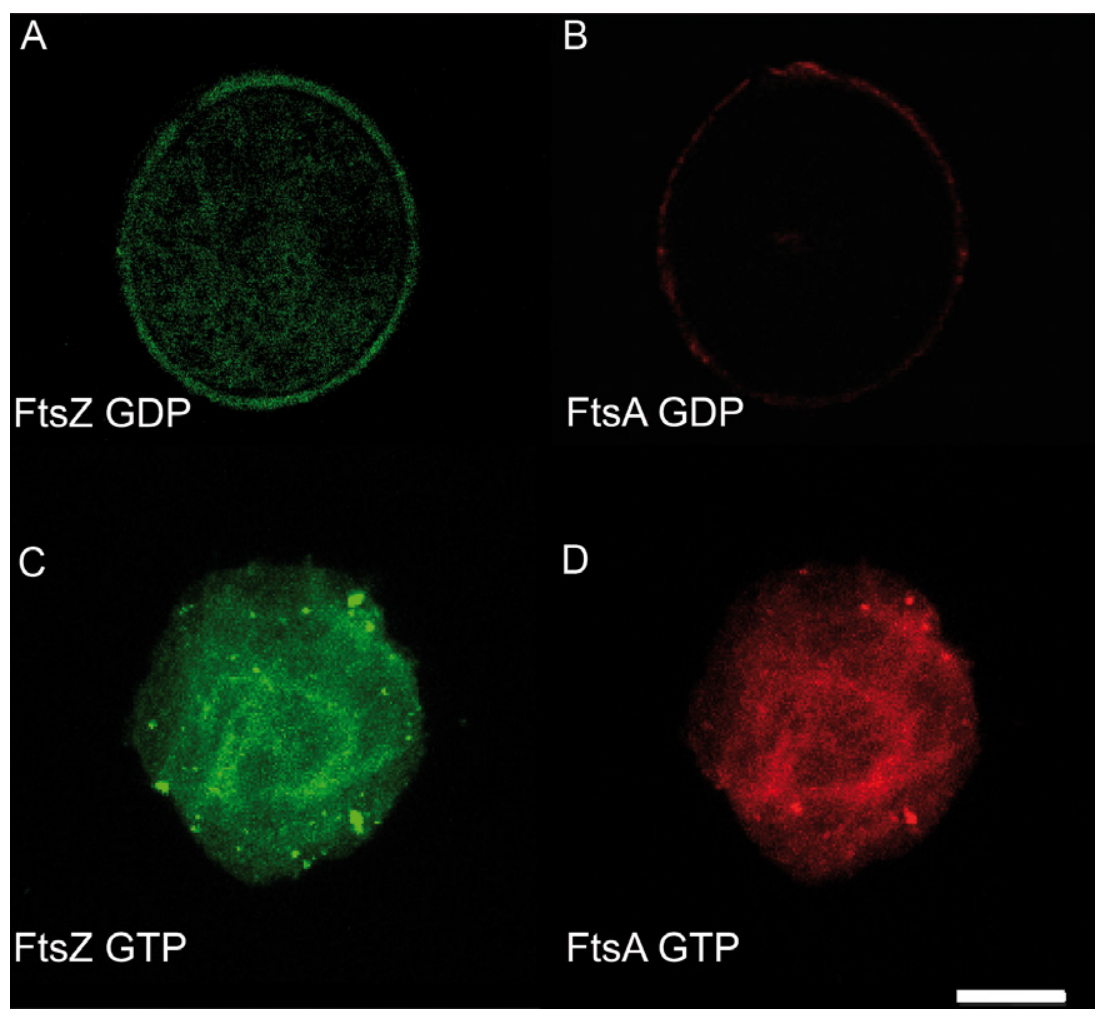

Fig. 5. Image analysis of FtsZ and FtsA inside Giant Unilamellar Inner Membrane Vesicles (GUIMVs) formed in the presence of $0.5 \mathrm{mM}$ caged GTP

A. Single equatorial sections of a GUIMV containing non-polymerized Alexa 488-labelled FtsZ.

B. Single equatorial sections of the same vesicle containing Alexa 647-labelled FtsA. C. Single equatorial sections of a GUIMV containing polymerized Alexa 488-labeled FtsZ.

D. Single equatorial sections of the same vesicle containing Alexa 647-labelled FtsA. Giant vesicles were obtained by electroformation from native inner membranes of $E$. coli in the presence of physiological salt concentration following the method described in Pott et al. 2008. Reprinted with permission from Jiménez and colleagues (2011). Bar represents $10 \mu \mathrm{m}$.

inside them. The application of these tools to study bacterial division elements and their interactions has been found to be feasible, as documented by several published experimental studies and many others currently in progress. However, despite these advances, it is clear that many experimental challenges need to be addressed before the division machinery can actually be reconstructed in the test tube, in the absence of cells (see also Martos et al., 2012a and Rivas et al., 2013). Optimal encapsulation of divisome elements in vesicles of proper size, shape and composition using permeable membranes and microfluidic technologies will be essential (Abkarian et al., 2011; Richmond et al., 2011; Takiguchi et al., 2011; Matosevic, 2012).

The constrictive force generator and constriction mechanism have to be reproduced in the test tube, which, among other things, would require refining the reconstitution experiments already done with a minimal set of division elements, to obtain very reproducible results under well-controlled experimental conditions. Membrane composition will also be relevant as it can directly influence the function of the incorporated proteins and the mechanical behaviour of the vesicles. This matters as the high protein content of the bacterial inner membrane provides different physical characteristics when compared with lipid membranes imposing specific mechanical constraints, which are likely to affect the perturbations associated to the force generated by FtsZ assembly/disassembly reactions. Moreover, membrane components redistributions during cell division should create lipid domains and regions highly concentrated in specific proteins that would favour the formation of curved structures and collaborate with force-generating protein machines, such as FtsZ, to constrict the bacterial cell membrane (Mileykovskaya and Dowhan, 2005). For these reasons, experimental studies using giant vesicles formed from whole or hybrid cell membranes (Merkle et al., 2008; Montes et al., 2010) will be instrumental to obtain biologically relevant observations.

The elements of the proto-ring complex may not be sufficient to obtain assemblies that perform activities that can be easily correlated with cell function. The incorporation of companion proteins could be required. Moreover, the action of site-selection systems (MinCDE complex and/or nucleoid occlusion) should be included. The latter relates with a crucial task: to control in time and space the organization of FtsZ networks in the vesicles, to specifically localize the anchoring of the polymers and eventually divide these cell-like containers. Besides these division-specific elements, non-specific factors, such as excluded volume effects and phase-separation processes (Hyman and Simons, 2012; Strulson et al., 2012), may play an important role to achieve this challenging goal. Along these lines, the incorporation of elements of the outer membrane and/or peptidoglycan elements (Egan and Vollmer, 2013) will be required to better reproduce the 
mechanical processes linked to bacterial division in the test tube.

Significant progress is expected by the application of cell-free expression systems to produce, in a controlled manner, division proteins inside giant vesicles, as recently achieved with MreB protein, which controls the morphology of rod-shaped bacteria (Maeda et al., 2012). These studies connect with research on the formation of protocells, in which these cell-like containers are excellent systems in bottom-up approaches to study how protocells divide as they can undergo shape-related changes that may result in self-production. In fact, morphological changes observed in giant vesicles are remarkably similar to those observed in bacterial L-forms wall-less cells (Briers et al., 2012; Errington, 2013; Mercier et al., 2013) reminiscent of primordial living organisms, suggesting some intriguing insights on the mechanisms of reproduction of primitive cells.

Finally, the work based on giant vesicle studies summarized above will need to be complemented with parallel studies using other cell-like containers, such as microdroplets (Theberge et al., 2010), droplet interface bilayers (Bayley et al., 2008) and polymersomes (Martino et al., 2012), together with powerful microfluidics technologies to reconstruct biologically relevant protein assemblies in their interior.

In summary, studies of specific division macromolecular complexes inside giant vesicles of well-defined composition will represent crucial steps towards reconstructing the minimal machinery required for dividing a cell-like membrane system. In addition these studies using minimal systems will help to evaluate the significance of these observations for cell function as they might be difficult to follow - or even hidden - within the extreme complexity of living cells. They must also be incorporated in molecular and cellular analysis aiming to develop quantitative global models of cellular processes. As the field of bottom-up synthetic biology is still emerging, front-line technologies such as the ones described here will serve to complete our knowledge in what ultimately will be considered a relevant aspect of integrative biology.

\section{Conflicts of interest}

The authors declare no conflicts of interest.

\section{Acknowledgements}

We thank Begoña Monterroso and Silvia Zorrilla (CIB) for critically reading the manuscript. This work was supported by the Spanish government through grants BIO2011-28941C03-03, by the European Commission through contract HEALTH-F3-2009-223432, and by Human Frontiers Science Program through grant RGP0050/2010-C102, all of them to GR.

\section{Note added in proof}

Procedures to incorporate FtsZ inside giant vesicles, formed by reverse emulsion, with ZipA artificially incorporated at the inner membrane face have recently been optimized (Cabré et al., 2013). These vesicles have been made permeable allowing the control of FtsZ polymerization inside them by externally added ligands that promote assembly, such as GTP and $\mathrm{Mg}^{2+}$. Mechanical effects of bacterial division have been reproduced in these permeable vesicles, which have also revealed in the cell. Interaction between FtsZ and ZipA affects both the morphology and the physiology of the membrane. Morphological changes observed in the vesicles and in the cell are compatible with pulling forces being exerted during constriction. In vitro, these forces may be modulated by the relative abundance of ZipA and by the dynamics of the FtsZ polymer. In vivo, an excess of ZipA results in multilayered membrane inclusions within the cytoplasm accompanied by the loss of the membrane function as a permeability barrier.

\section{References}

Abkarian, M., Loiseau, E., and Massiera, G. (2011) Continuous droplet interface crossing encapsulation (cDICE) for high throughput monodisperse vesicle design. Softmatter 7: 4610-4614.

Adams, D.W., and Errington, J. (2009) Bacterial cell division: assembly, maintenance and disassembly of the $\mathrm{Z}$ ring. Nat Rev Microbiol 7: 642-653.

Angelova, M.I., and Dimitrov, D.D. (1986) Liposome electroformation. Faraday Discuss Chem Soc 81: 303311.

Angelova, M.I., Soleau, S., Meleard, P., Faucon, J.F., and Bothorel, P. (1992) Preparation of giant vesicles by external AC electric fields. Kinetics and applications. Prog Colloid Polym Sci 89: 127-131.

Baumbart, T., Caprarp, B.R., Zhu, C.G., and Das, S.L. (2011) Thermodynamics and mechanics of membrane curvature generation and sensing by proteins and lipids. Annu Rev Phys Chem 62: 483-506.

Bayley, H., Cronin, B., Heron, A., Holden, M.A., Hwang, W.L., Syeda, R., et al. (2008) Droplet interface bilayers. Mol Biosyst 4: 1191-1208.

Briers, Y., Walde, P., Schuppler, M., and Loessner, M.J. (2012) How did bacterial ancestors reproduce? Lessons from L-form cells and giant lipid vesicles: multiplication similarities between lipid vesicles and L-form bacteria. Bioessays 34: 1078-1084.

Cabré, E.J., Sánchez-Gorostiaga, A., Carrara, P., Ropero, N., Casanova, M., Palacios, P., et al. (2013) Bacterial division proteins FtsZ and ZipA induce vesicle shrinkage and cell membrane invagination. J Biol Chem. doi: 10.1074/ jbc.M113.491688

Carrara, P., Stano, P., and Luisi, P.L. (2012) Giant vesicles 'colonies': a model for primitive cell communities. Chembiochem 13: 1497-1502. 
Dominak, L.M., and Keating, C.D. (2008) Macromolecular crowding improves polymer encapsulation within giant lipid vesicles. Langmuir 24: 13565-13571.

Egan, A.J., and Vollmer, W. (2013) The physiology of bacterial cell division. Ann N Y Acad Sci 1277: 8-28.

Endy, D. (2005) Foundations for engineering biology. Nature 438: 449-453.

Erickson, H.P., Anderson, D.E., and Osawa, M. (2010) FtsZ in bacterial cytokinesis: cytoskeleton and force generator all in one. Microbiol Mol Biol Rev 74: 504-528.

Errington, J. (2013) L-form bacteria, cell walls and the origins of life. Open Biol 3: 120143. doi:10.1098/rsob.120143.

González, J.M., Jiménez, M., Velez, M., Mingorance, J., Andreu, J.M., Vicente, M., and Rivas, G. (2003) Essential cell division protein FtsZ assembles into one monomerthick ribbons under conditions resembling the crowded intracellular environment. J Biol Chem 278: 37664-37671.

Horger, K.S., Estes, D.J., Capone, R., and Mayer, M. (2009) Films of agarose enable rapid formation of giant liposomes in solutions of physiologic ionic strength. J Am Chem Soc 131: 1810-1819.

Hyman, A.A., and Simons, K. (2012) Cell biology. Beyond oil and water - phase transitions in cells. Science 337: 10471049.

Jiménez, M., Martos, A., Vicente, M., and Rivas, G. (2011) Reconstitution and organization of Escherichia coli protoring elements (FtsZ and FtsA) inside giant unilamellar vesicles obtained from bacterial inner membranes. J Biol Chem 286: 11236-11241.

Kale, S.D., Gu, B., Capelluto, D.G., Dou, D., Feldman, E., Rumore, A., et al. (2010) External lipid PI3P mediates entry of eukaryotic pathogen effectors into plant and animal host cells. Cell 142: 284-295.

Kuroiwa, T., Fujita, R., Kobayashi, I., Uemura, K., Nakajima, M., Sato, S., et al. (2012) Efficient preparation of giant vesicles as biomimetic compartment systems with high entrapment yields for biomacromolecules. Chem Biodivers 9: 2453-2472.

Li, T.H., Stachowiak, J.C., and Fletcher, D.A. (2009) Mixing solutions in inkjet formed vesicles. Methods Enzymol 465: 75-94.

Liu, A.P., and Fletcher, D.A. (2009) Biology under construction: in vitro reconstitution of cellular function. Nat Rev Mol Cell Biol 10: 644-650.

Liu, A.P., Richmond, D.L., Maibaum, L., Pronk, S., Geissler, P.L., and Fletcher, D.A. (2008) Membrane-induced bundling of actin filaments. Nat Phys 4: 789-793.

Lopez-Montero, I., Lopez-Navajas, P., Mingorance, J., Velez, M., Vicente, M., and Monroy, F. (2013) Membrane reconstitution of FtsZ-ZipA complex inside giant spherical vesicles made of $\mathrm{E}$. coli lipids: large membrane dilation and analysis of membrane plasticity. Biochim Biophys Acta 1828: 687-698.

Lutkenhaus, J., Pichoff, S., and Du, S. (2012) Bacterial cytokinesis: from $\mathrm{Z}$ ring to divisome. Cytoskeleton (Hoboken) 69: 778-790.

Maeda, Y.T., Nakadai, T., Shin, J., Uryu, K., Noireaux, V., and Libchaber, A. (2012) Assembly of MreB filaments on liposome membranes: a synthetic biology approach. ACS Synth Biol 1: 53-59.

Martino, C., Kim, S.H., Horsfall, L., Abbaspourrad, A.,
Rosser, S.J., Cooper, J., and Weitz, D.A. (2012) Protein expression, aggregation, and triggered release from polymersomes as artificial cell-like structures. Angew Chem Int Ed Engl 51: 6416-6420.

Martos, A., Jiménez, M., Rivas, G., and Schwille, P. (2012a) Towards a bottom-up reconstitution of bacterial cell division. Trends Cell Biol 22: 634-643.

Martos, A., Monterroso, B., Zorrilla, S., Reija, B., Alfonso, C., Mingorance, J., et al. (2012b) Isolation, characterization and lipid-binding properties of the recalcitrant FtsA division protein from Escherichia coli. PLOS ONE 7: e39829.

Matosevic, S. (2012) Synthesizing artificial cells from giant unilamellar vesicles: state-of-the art in the development of microfluidic technology. Bioessays 34: 992-1001.

Mercier, R., Kawai, Y., and Errington, J. (2013) Excess membrane synthesis drives a primitive mode of cell proliferation. Cell 152: 997-1007.

Merkle, D., Kahya, N., and Schwille, P. (2008) Reconstitution and anchoring of cytoskeleton inside giant unilamellar vesicles. Chembiochem 9: 2673-2681.

Milam, S.L., Osawa, M., and Erickson, H.P. (2012) Negativestain electron microscopy of inside-out FtsZ rings reconstituted on artificial membrane tubules show ribbons of protofilaments. Biophys J 103: 59-68.

Mileykovskaya, E., and Dowhan, W. (2005) Role of membrane lipids in bacterial division-site selection. Curr Opin Microbiol 8: 135-142.

Mingorance, J., Rivas, G., Velez, M., Gomez-Puertas, P., and Vicente, M. (2010) Strong FtsZ is with the force: mechanisms to constrict bacteria. Trends Microbiol 18: 348-356.

Monterroso, B., Alfonso, C., Zorrilla, S., and Rivas, G. (2013) Combined analytical ultracentrifugation, light scattering and fluorescence spectroscopy studies on the functional associations of the bacterial division FtsZ protein. Methods 59: 349-362.

Montes, L.R., Ahyayauch, H., Ibarguren, M., Sot, J., Alonso, A., Bagatolli, L.A., and Goni, F.M. (2010) Electroformation of giant unilamellar vesicles from native membranes and organic lipid mixtures for the study of lipid domains under physiological ionic-strength conditions. Methods $\mathrm{Mol}$ Biol 606: 105-114.

Nandagopal, N., and Elowitz, M.B. (2011) Synthetic biology: integrated gene circuits. Science 333: 1244-1248.

Noireaux, V., Maeda, Y.T., and Libchaber, A. (2011) Development of an artificial cell, from self-organization to computation and self-reproduction. Proc Natl Acad Sci USA 108: 3473-3480.

Osawa, M., and Erickson, H.P. (2011) Inside-out Z rings constriction with and without GTP hydrolysis. Mol Microbiol 81: 571-579.

Osawa, M., Anderson, D.E., and Erickson, H.P. (2008) Reconstitution of contractile FtsZ rings in liposomes. Science 320: 792-794.

Osawa, M., Anderson, D.E., and Erickson, H.P. (2009) Curved FtsZ protofilaments generate bending forces on liposome membranes. EMBO J 28: 3476-3484.

Ota, S., Yoshizawa, S., and Takeuchi, S. (2009) Microfluidic formation of monodisperse, cell-sized, and unilamellar vesicles. Angew Chem Int Ed Engl 48: 6533-6537.

Pautot, S., Frisken, B.J., and Weitz, D.A. (2003) Engineering 
asymmetric vesicles. Proc Natl Acad Sci USA 100: 1071810721.

Pott, T., Bouvrais, H., and Meleard, P. (2008) Giant unilamellar vesicle formation under physiologically relevant conditions. Chem Phys Lipids 154: 115-119.

Reeves, J.P., and Dowben, R.M. (1969) Formation and properties of thin-walled phospholipid vesicles. J Cell Physiol 73: 49-60.

Richmond, D.L., Schmid, E.M., Martens, S., Stachowiak, J.C., Liska, N., and Fletcher, D.A. (2011) Forming giant vesicles with controlled membrane composition, asymmetry, and contents. Proc Natl Acad Sci USA 108: 94319436.

Rico, A.I., Garcia-Ovalle, M., Palacios, P., Casanova, M., and Vicente, M. (2010) Role of Escherichia coli FtsN protein in the assembly and stability of the cell division ring. Mol Microbiol 76: 760-771.

Rivas, G., Alfonso, C., Jiménez, M., Monterroso, B., and Zorrilla, S. (2013) Macromolecular interactions of the bacterial division FtsZ protein: from quantitative biochemistry and crowding to reconstructing minimal divisomes in the test tube. Biophys Rev 5: 63-77.

Schwille, P. (2011) Giant unilamellar vesicles: from minimal membrane systems to minimal cells? In The Minimal Cell: The Biophysics of Cell Compartment and the Origin of Cell Functionality. Stano, L.A. (ed.). Heidelberg, Germany: Springer Science+Business Media B. V, pp. 231-253.

Schwille, P. (2013) Editorial: chemistry needed: synthetic biology as a new incentive for interdisciplinarity. Angew Chem Int Ed Engl 52: 2616-2617.

Schwille, P., and Diez, S. (2009) Synthetic biology of minimal systems. Crit Rev Biochem Mol Biol 44: 223242.

Stachowiak, J.C., Richmond, D.L., Li, T.H., Liu, A.P., Parekh, S.H., and Fletcher, D.A. (2008) Unilamellar vesicle formation and encapsulation by microfluidic jetting. Proc Natl Acad Sci USA 105: 4697-4702.

Stachowiak, J.C., Hayden, C.C., and Sasaki, D.Y. (2010) Steric confinement of proteins on lipid membranes can drive curvature and tubulation. Proc Natl Acad Sci USA 107: 7781-7786.

Stachowiak, J.C., Schmid, E.M., Ryan, C.J., Ann, H.S.,
Sasaki, D.Y., Sherman, M.B., et al. (2012) Membrane bending by protein-protein crowding. Nat Cell Biol 14: 944949.

Stano, P., and Luisi, P.L. (2013) Semi-synthetic minimal cells: origin and recent developments. Curr Opin Biotechnol 24: $1-6$.

Strulson, C.A., Molden, R.C., Keating, C.D., and Bevilacqua, P.C. (2012) RNA catalysis through compartmentalization. Nat Chem 4: 941-946.

Sugiura, S., Kuroiwa, T., Kagota, T., Nakajima, M., Sato, S., Mukataka, S., et al. (2008) Novel method for obtaining homogeneous giant vesicles from a monodisperse waterin-oil emulsion prepared with a microfluidic device. Langmuir 24: 4581-4588.

Takiguchi, K., Negishi, M., Tanaka-Takiguchi, Y., Homma, M., and Yoshikawa, K. (2011) Transformation of actoHMM assembly confined in cell-sized liposome. Langmuir 27: 11528-11535.

Terasawa, H., Nishimura, K., Suzuki, H., Matsuura, T., and Yomo, T. (2013) Coupling of the fusion and budding of giant phospholipid vesicles containing macromolecules. Proc Natl Acad Sci USA 109: 5942-5947.

Theberge, A.B., Courtois, F., Schaerli, Y., Fischlechner, M., Abell, C., Hollfelder, F., and Huck, W.T. (2010) Microdroplets in microfluidics: an evolving platform for discoveries in chemistry and biology. Angew Chem Int Ed Engl 49: 5846-5868.

Tsai, F.C., Stuhrmann, B., and Koenderink, G.H. (2011) Encapsulation of active cytoskeletal protein networks in cell-sized liposomes. Langmuir 27: 10061-10071.

Vicente, M., and Rico, A.I. (2006) The order of the ring: assembly of Escherichia coli cell division components. Mol Microbiol 61: 5-8.

Vicente, M., Rico, A.I., Martinez-Arteaga, R., and Mingorance, J. (2006) Septum enlightenment: assembly of bacterial division proteins. J Bacteriol 188: 19-27.

Walde, P., Cosentino, K., Engel, H., and Stano, P. (2010) Giant vesicles: preparations and applications. Chembiochem 11: 848-865.

Zhou, H.X., Rivas, G., and Minton, A.P. (2008) Macromolecular crowding and confinement: biochemical, biophysical, and potential physiological consequences. Annu Rev Biophys 37: 375-397. 